\title{
REDUÇÃO DE DOSE E AUMENTO NA VIDA ÚTIL DO TUBO DE RAIOS X EM TOMOGRAFIA COMPUTADORIZADA*
}

\author{
José Augusto Marconato ${ }^{1}$, Alessandro A. Mazzola ${ }^{2}$, Paulo César Sanvitto ${ }^{3}$, \\ Antonio Carlos Pires Carvalho ${ }^{4}$, Márcio Vieveger Vasques ${ }^{1}$
}

Resumo OBJETIVO: Reduzir a dose de radiação e aumentar a vida útil do tubo de raios $X$ em exames de tomografia computadorizada. MATERIAIS E MÉTODOS: Foram avaliados exames de crânio, abdome superior e tórax. Foi verificado se a técnica utilizada poderia ser alterada, foram sugeridos novos protocolos, e feitas comparações de qualidade da imagem, dose de radiação e aquecimento do tubo de raios X. RESULTADOS: Uma redução no mAs pôde ser feita sem comprometer a qualidade do diagnóstico, proporcionando redução de até $20 \%$ na dose média dos exames de crânio em adultos e de até $45 \%$ em crianças com idade de 0 a 6 meses; pacientes com menos de $50 \mathrm{~kg}$ tiveram redução de aproximadamente $37 \%$ na dose média de radiação para os exames de abdome superior; para o exame de tórax de rotina a redução chegou a $54 \%$. 0 aquecimento do tubo de raios $X$ para os exames de crânio, abdome superior e tórax teve redução estimada em aproximadamente $13 \%, 23 \%$ e $41 \%$, respectivamente. CONCLUSÃO: Uma alteração nos protocolos dos exames descritos acarretará diminuição significativa na dose de radiação e aumento na vida útil do tubo de raios $X$, sem comprometer o diagnóstico.

Unitermos: Tomografia computadorizada por raios $\mathrm{X}$; Exposição à radiação; Dosagem de radiação; Proteção radiológica.

Abstract Dose reduction and increase of X-ray tube life in computed tomography.

OBJECTIVE: To reduce radiation dose and increase $x$-ray tube life in computed tomography. MATERIALS AND METHODS: Head, upper abdomen and chest scans were evaluated. We assessed the techniques employed and if these could be changed, suggested new protocols and compared the quality of the images, the radiation dose and the x-ray tube heating. RESULTS: A mAs reduction could be done without interfering with the diagnostic quality, allowing a decrease of up to $20 \%$ in the average dose for adults' head exams and up to $45 \%$ for 0 to 6 years old children; patients with less than $\mathbf{5 0} \mathbf{~ k g ~ h a d ~ a ~ r e d u c t i o n ~ o f ~}$ $37 \%$ on the upper abdomen radiation average dose; for chest routine scans the reduction was $54 \%$. The decrease in the heating of the $x$-ray tube for head, upper abdomen and chest scans was approximately $13 \%$, $23 \%$ and $41 \%$, respectively. CONCLUSION: A change on the described protocols will produce a significant reduction on the radiation dose and an increasing in $x$-ray tube life without loss of diagnostic quality. Key words: X-ray computed tomography; Radiation exposure; Radiation dosage; Radiation protection.

\section{INTRODUÇÃO}

Desde a introdução da tomografia computadorizada (TC) na prática clínica em 1972, tem ocorrido grande aumento no número de exames realizados ${ }^{(\mathbf{1 , 2})}$, acarretando um aumento significativo na dose de radiação recebida pela população ${ }^{(3)}$. Além dis-

* Trabalho realizado no Hospital Moinhos de Vento, Porto Alegre, RS.

1. Serviço de Física Médica do Hospital Moinhos de Vento.

2. Serviço de Física Médica do Hospital Moinhos de Vento, Faculdade de Física da Pontifícia Universidade Católica do Rio Grande do Sul (PUCRS), Centro de Educação Tecnológica do Instituto Pró-Universidade Canoense (Cetipuc).

3. Radiologista do Hospital Moinhos de Vento.

4. Faculdade de Medicina da Universidade Federal do Rio de Janeiro (UFRJ).

Endereço para correspondência: José Augusto Marconato. Rua Marquês do Herval, 65/302, Bairro Marechal Rondon. Canoas, RS, 92020-400. E-mail: ressonancia@hmv.org.br

Recebido para publicação em 18/7/2003. Aceito, após revisão, em 3/10/2003. so, a dose absorvida por crianças é maior do que em pacientes adultos ${ }^{(\mathbf{4})}$. Em qualquer aquisição de TC, para um potencial de tubo e espessura de corte constantes, a dose de radiação no paciente varia proporcionalmente com a carga transportável (mAs) selecionada pelo operador. A escolha do mAs também determina a quantidade de ruído na imagem ${ }^{(5,6)}$ e o aquecimento no tubo de raios $\mathrm{X}^{(7)}$. Estudos mostraram que o mAs utilizado pode ser reduzido consideravelmente sem prejudicar o diagnóstico, principalmente para exames de tórax ${ }^{(8-10)}$ e em crianças $^{(11)}$. A redução no aquecimento do tubo de raios $\mathrm{X}$ ocasiona um menor desgaste, fazendo com que mais exames possam ser realizados com a mesma ampola. Os gastos relacionados à troca deste componente são elevados, o seu valor pode superar 50 mil dólares e a periodicidade pode ser menor que anual. Esses dois aspectos importantes na TC, a dose de radiação e o desgaste do tubo de raios $\mathrm{X}$, estão diretamente ligados, pois ao reduzirmos a dose estamos reduzindo o desgaste do tubo. Dessa forma, se faz necessária a realização de um estudo desses dois fatores para que possa ser aplicada uma otimização nos protocolos utilizados nos exames de TC.

O objetivo deste trabalho é verificar os protocolos utilizados nos exames mais realizados no serviço e sugerir uma redução no mAs utilizado, comparando a dose de radiação, o aquecimento do tubo de raios $\mathrm{X}$ e a qualidade da imagem.

\section{MATERIAIS E MÉTODOS}

Este trabalho foi desenvolvido utilizando-se um equipamento da marca General 
Electric (GE) modelo Prospeed ${ }^{\circledR}$ de um hospital privado.

Estão descritas, a seguir, as etapas seguidas na realização do estudo:

a) seleção dos exames, na qual as informações sobre o número e o tipo de procedimentos realizados durante um ano foram obtidas por meio de uma pesquisa no arquivo do sistema informatizado do serviço;

b) pesquisa dos parâmetros técnicos utilizados, realizada com o auxílio do "software" eFilm Workstation 1.5.3 ${ }^{(\mathbf{1 2})}$. Os dados foram coletados de janeiro a abril de 2002, de acordo com o sexo, idade, peso, tensão, carga transportável, espessura e número de cortes, intervalo entre eles e área de cobertura anatômica;

c) estudo de uma possível alteração no mAs utilizado, com a finalidade de redução de dose no paciente e aumento na vida útil do tubo de raios $\mathrm{X}$;

d) avaliação em um simulador padrão de qualidade da imagem, quanto ao ruído (com medidas em três posições - 12h, $3 \mathrm{~h}$ e 6h) e resolução de baixo contraste;

e) aplicação do novo protocolo em apenas uma secção do paciente;

f) análise da imagem resultante, sem visualização da técnica utilizada, e resposta de um questionário pelos médicos radiologistas responsáveis pela interpretação de cada tipo de exame;

g) medida comparativa da dose média em múltiplos cortes (MSAD) com os parâmetros técnicos originais e com os protocolos propostos, utilizando-se simuladores de cabeça e corpo recomendados pela American Association of Physicists in Medicine $^{(\mathbf{1 3})}$ (Figura 1) e câmara de ionização em forma de lápis, modelo 10X5-3CT (Radcal Corporation ${ }^{\circledR}$ ) (Figura 2A), com comprimento efetivo de $100 \mathrm{~mm}$ acoplada

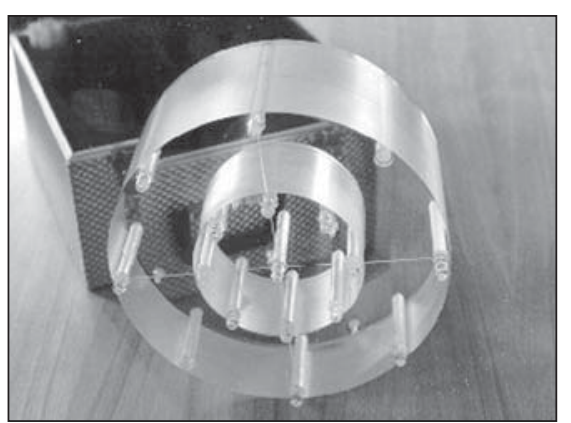

Figura 1. Simulador de acrílico para medida de MSAD em exames de crânio e corpo. a um monitor de radiação modelo 9015 (Radcal Corporation $^{\circledR}$ ) (Figura 2B);

h) cálculo comparativo do aquecimento do tubo de raios $\mathrm{X}$, em unidades de calor (HU), com os dois protocolos. O cálculo foi realizado utilizando-se a equação:

$$
H U=k V . m A \cdot t . n
$$

onde: $k V$ é a tensão; $m A$, a corrente; $t$, o tempo de exposição; $n$, o número de cortes utilizados.

\section{RESULTADOS}

\section{Seleção dos exames mais realizados}

A Tabela 1 apresenta os exames selecionados e seu porcentual em relação ao total realizado no ano de 2001.

Os exames de crânio, abdome superior e tórax foram selecionados para estudo por representarem $57,9 \%$ da totalidade dos procedimentos realizados.

\section{Verificação da técnica utilizada em cada exame, sugestão de novos protocolos e avaliação da qualidade da imagem}

\section{Exames de crânio}

Foram avaliados 40 exames. Como mostra a Figura 3, não havia relação do mAs utilizado com a idade do paciente, uma vez que foi empregado o mesmo mAs para crianças e para adultos, quando o esperado era que fossem utilizados valores mais baixos. Também se observou variação de até $120 \mathrm{mAs}$ para pacientes adultos com a mesma idade. Constatou-se que, na sua maioria, os exames foram realizados com

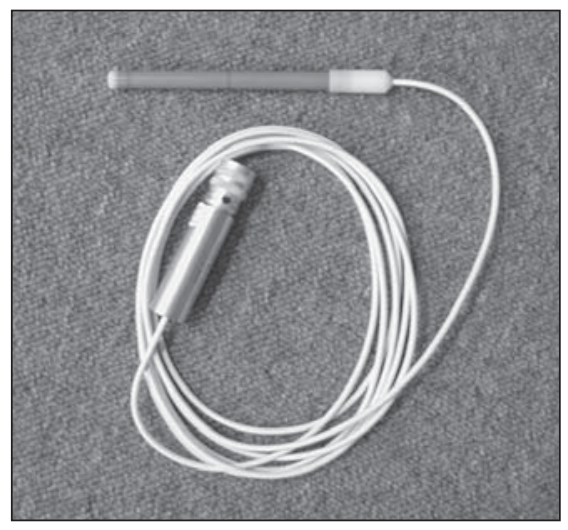

A

Figura 2. A: Câmara de ionização em forma de lápis utilizada para medida de dose em TC. B: Monitor de radiação.
Tabela 1 Tipo, quantidade e porcentual dos exames selecionados em relação ao total realizado em 2001.

\begin{tabular}{|l|c|c|}
\hline \multicolumn{1}{|c|}{ Tipo de exame } & № de exames & \% Total \\
\hline TC crânio & 1.593 & $20,6 \%$ \\
TC abdome superior & 1.579 & $20,4 \%$ \\
TC tórax & 1.312 & $16,9 \%$ \\
\hline
\end{tabular}

320 mAs na região da fossa posterior e 260 mAs na região supratentorial. Em alguns casos o mAs foi reduzido para 260 e 200 nestas regiões, respectivamente, e em outros, utilizado $320 \mathrm{mAs}$ para ambas. Em crianças, o mAs utilizado foi de 260 para a região da fossa posterior e de 200 para a região supratentorial. Em um exame foi utilizado $260 \mathrm{mAs}$ para as duas regiões.

Como já se realizavam alguns exames com mAs reduzido, sugeriu-se uma padronização para todos os exames de pacientes adultos, ou seja, a utilização de 260 mAs na região da fossa posterior (3 $\mathrm{mm}$ de espessura de corte) e $200 \mathrm{mAs}$ na região supratentorial (5 $\mathrm{mm}$ de espessura de corte).

A Tabela 2 mostra os valores de ruído e número de orifícios de baixo contraste visualizados em simulador padrão, comparando as duas técnicas de exame.

Como era esperado, ocorreu aumento do ruído com a diminuição do mAs utilizado, mas este aumento não reduziu a visualização dos orifícios de baixo contraste (Figura 4). Neste caso não foi necessária a aquisição de imagem comparativa, uma vez que o protocolo com mAs reduzido já havia sido utilizado. Os médicos radiologistas concordaram que um pequeno aumento

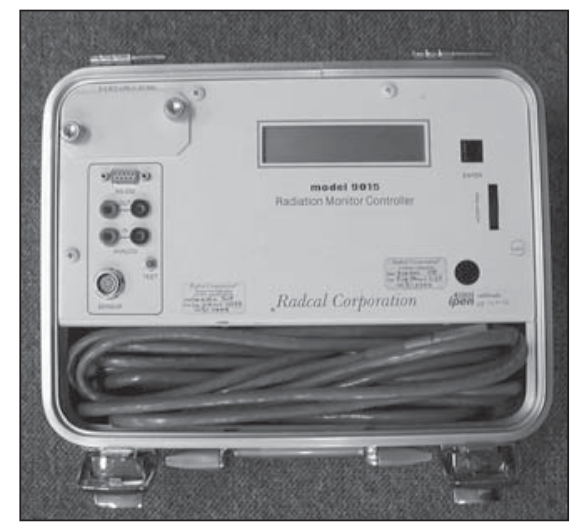
B 
Figura 3. Relação entre o mAs e a idade do paciente em exames de crânio.

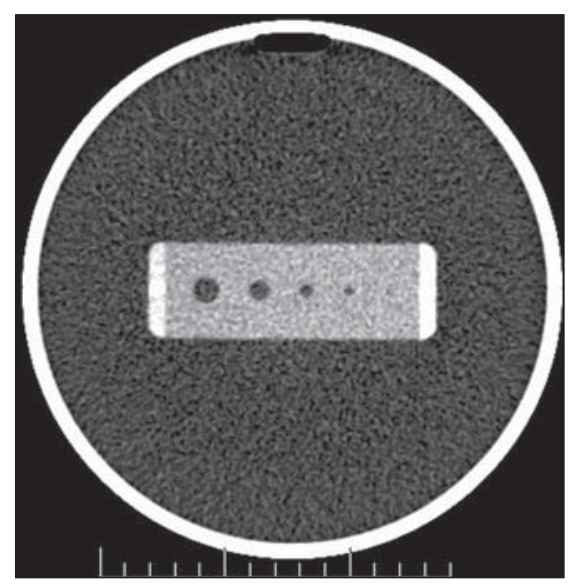

A
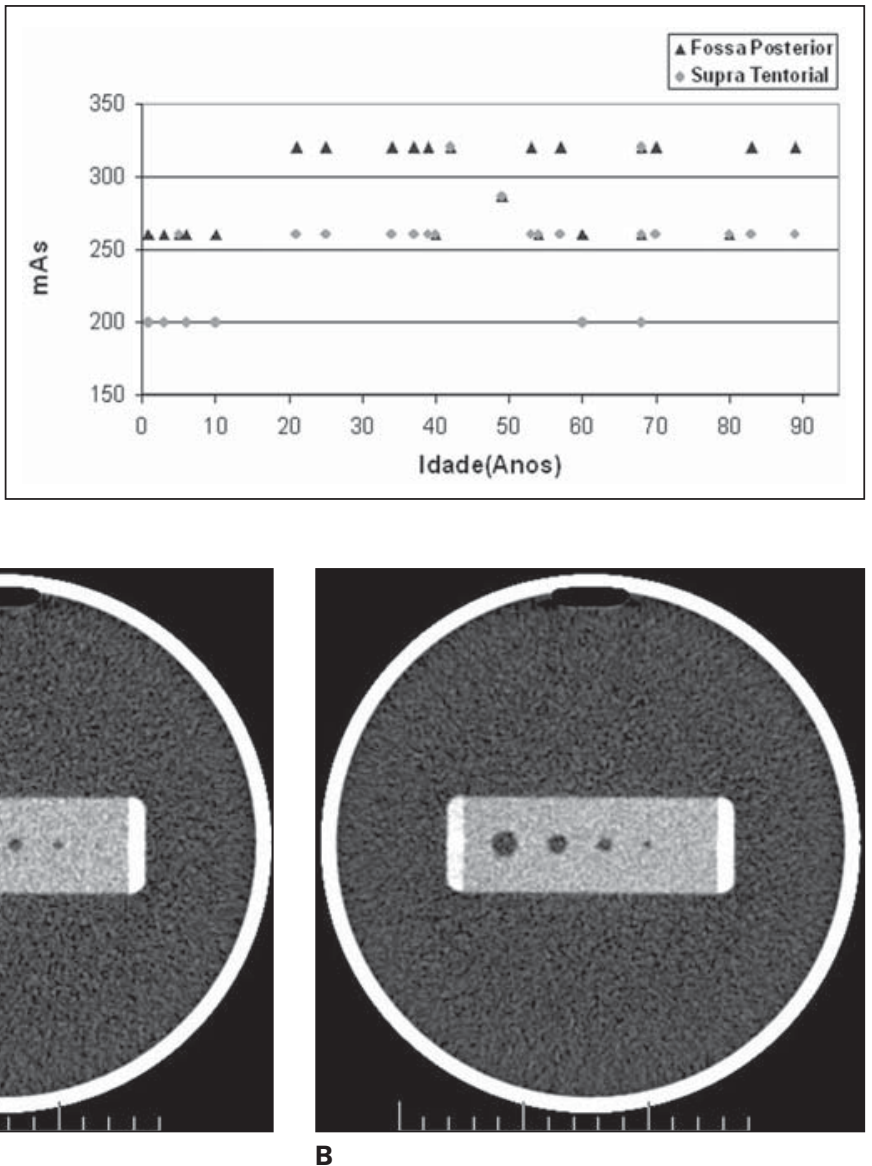

Figura 4. Imagens do simulador com 3 mm de espessura de corte. A: 260 mAs; B: 320 mAs.

Tabela 2 Medidas de ruído e número de orifícios de baixo contraste visualizados no simulador padrão.

\begin{tabular}{|c|c|c|c|c|}
\hline mAs & Espessura de corte & Região & № de orifícios & Ruído médio \\
\hline 200 & $5 \mathrm{~mm}$ & Supratentorial & 4 & $3,4 \pm 0,3$ \\
260 & $5 \mathrm{~mm}$ & Supratentorial & 4 & $2,9 \pm 0,06$ \\
260 & $3 \mathrm{~mm}$ & Fossa posterior & 4 & $3,5 \pm 0,1$ \\
320 & $3 \mathrm{~mm}$ & Fossa posterior & 4 & $4,0 \pm 0,1$ \\
\hline
\end{tabular}

no ruído da imagem não prejudicou, neste caso, a qualidade do diagnóstico.

Para pacientes pediátricos sugere-se redução no mAs conforme a faixa etária em que estes se enquadram, pois o diâmetro e a densidade do crânio são proporcionais à idade desses pacientes. Para crianças acima de dez anos sugere-se o mesmo protocolo utilizado em adultos.

A Tabela 3 apresenta os valores de mAs sugeridos para os exames de crânio em crianças, de acordo com a faixa etária.

As medidas de ruído apresentaram aumento gradativo em seus valores com a diminuição do mAs utilizado. Mesmo assim, pôde-se visualizar quatro orifícios de
Tabela 3 Valores de mAs sugeridos para exames de crânio em crianças conforme a faixa etária.

\begin{tabular}{|c|l|r|}
\hline Pacientes & \multicolumn{1}{|c|}{ Região } & mAs \\
\hline \multirow{2}{*}{ 5-10 anos } & Fossa posterior & 240 \\
& Supratentorial & 195 \\
3-5 anos & Fossa posterior & 200 \\
& Supratentorial & 195 \\
1-3 anos & Fossa posterior & 195 \\
& Supratentorial & 160 \\
6 meses-1 ano & Fossa posterior & 130 \\
& Supratentorial & 120 \\
0-6 meses & Fossa posterior & 100 \\
& Supratentorial & 90 \\
\hline
\end{tabular}

Para este tipo de exame foi relacionado o peso e não a idade do paciente com o mAs utilizado, pois, geralmente, pacientes com peso mais elevado possuem diâmetro maior do abdome.

Pôde-se observar que pacientes com mesmo peso realizaram o exame com valores bem diferentes de mAs, e pacientes com pesos bastante diferentes realizaram o exame com o mesmo valor de mAs. Além disso, apesar de ter-se coletado dados de apenas uma criança, constatou-se que o protocolo utilizado foi o mesmo que para adultos. Sugere-se a utilização de valores de mAs que levem em consideração o peso do paciente, ou seja, adultos de até $50 \mathrm{~kg}$, $200 \mathrm{mAs}$, de 50 a $80 \mathrm{~kg}, 260 \mathrm{mAs}$ e acima de $80 \mathrm{~kg}, 320$ mAs. O aumento do ruído acaba sendo compensado pela diminuição do diâmetro do paciente.

As imagens do simulador permitiram visualizar quatro orifícios de baixo contraste para todos os valores de mAs. Como já se utilizava 260 mAs em alguns pacientes, foram realizados cortes comparativos apenas com a utilização de 200 mAs e 320 $\mathrm{mAs}$ em um paciente com peso inferior a $50 \mathrm{~kg}$. As Figuras 6A e 6B foram apresentadas aos radiologistas, que não observaram redução significativa na qualidade da imagem, aprovando os novos parâmetros.

Por terem sido coletados dados de apenas uma criança, os protocolos foram definidos somente para pacientes adultos. Em crianças, portanto, podem ser utilizados valores ainda menores de mAs.

\section{Exames de tórax}

Foram avaliados 30 exames. Estes foram realizados com três protocolos diferentes. Para imagens de alta resolução foi 


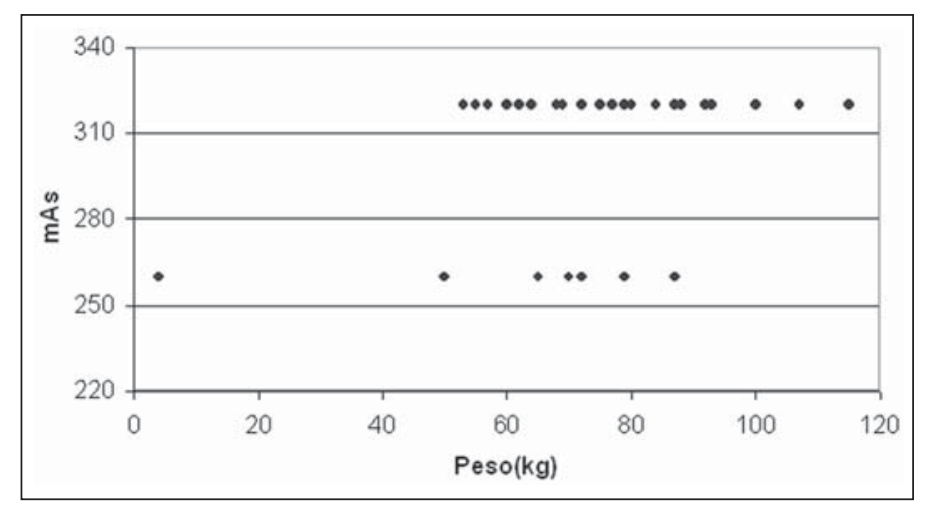

Figura 5. Relação entre o mAs utilizado e o peso do paciente em exames de abdome superior.

utilizado $260 \mathrm{mAs}$ com espessura de corte de $1 \mathrm{~mm}$ em aquisição seqüencial; para exame de rotina, $260 \mathrm{mAs}$ com $5 \mathrm{~mm}$ de espessura de corte em aquisição seqüencial; e 195 mAs com 10 mm de espessura de corte para aquisição helicoidal.

Como pode ser observado nas Figuras 7 e 8, existe uma padronização na realização dos exames avaliados com $260 \mathrm{mAs}$ para espessuras de corte de 1 e $5 \mathrm{~mm}$ em aquisição seqüencial e 195 mAs para espessuras de $10 \mathrm{~mm}$ em aquisição helicoi- dal em pacientes adultos. Apenas um paciente realizou exame com 320 mAs.

Como comentado anteriormente, os exames de tórax seguem um padrão; entretanto, por ser uma região do corpo que absorve pouca radiação, é possível reduzir o mAs para todas as espessuras de corte utilizadas. Ravenel et al. $^{(\mathbf{8})}$ mostraram que é possível uma redução para até 120 de tórax sem prejudicar a qualidade do diagnóstico, exceto em alta resolução. mAs na técnica tomográfica para exames
Sugere-se a alteração dos protocolos de adultos reduzindo-se para 120 o mAs utilizado nos exames seqüenciais de rotina com $5 \mathrm{~mm}$ e helicoidal com $10 \mathrm{~mm}$ de espessura de corte. Para os exames de alta resolução sugere-se $150 \mathrm{mAs}$.

Foram definidos protocolos apenas para pacientes adultos, pois não obtivemos dados de crianças que realizaram esse exame. Entretanto, podem ser utilizados valores ainda menores de mAs nesses casos.

Os médicos radiologistas aprovaram as imagens com $\mathrm{mAs}$ reduzido ao compararem com as de alto valor de mAs. As Figuras 9A e 9B mostram as imagens obtidas com 120 mAs, em exame de rotina, e as Figuras 10A e $10 \mathrm{~B}$ as imagens obtidas com $150 \mathrm{mAs}$ em exame com protocolo de alta resolução.

\section{Medidas comparativas de dose} e aquecimento do tubo de raios $X$

\section{Exames de crânio}

$\mathrm{O}$ resultado das medidas comparativas de MSAD e a sua redução porcentual levando em consideração a diminuição su-

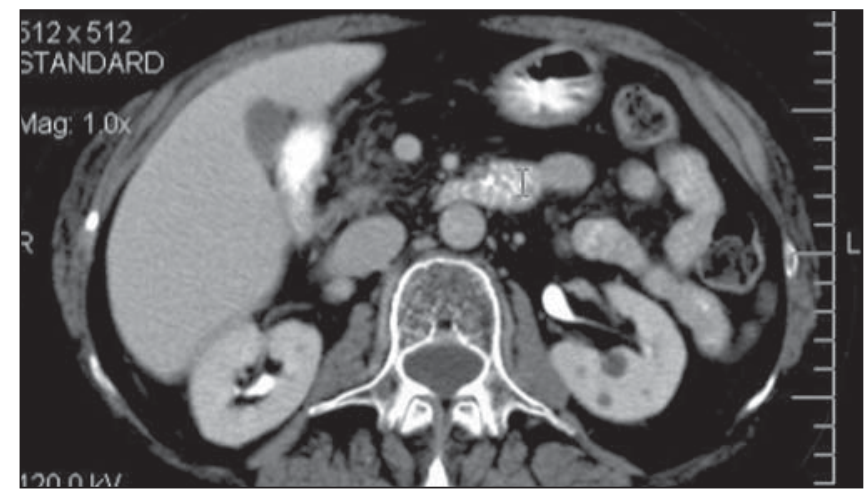

A

Figura 6. Imagem do abdome com 5 mm de espessura de corte. A: 200 mAs; B: 320 mAs.

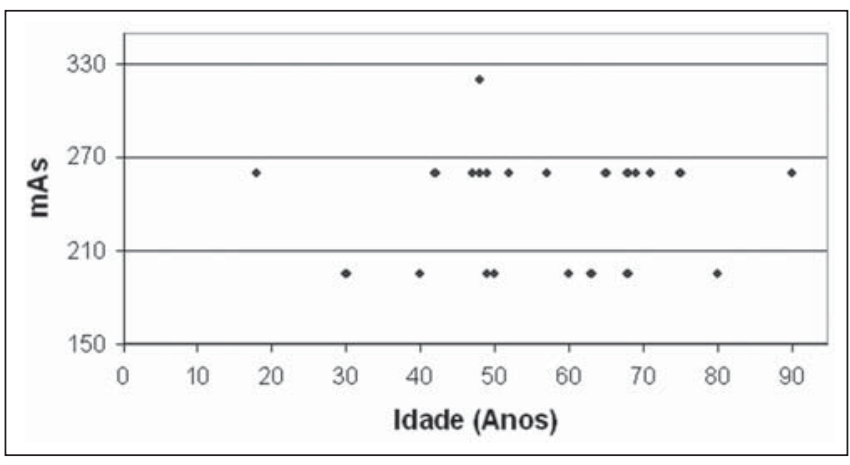

Figura 7. Relação entre o mAs utilizado e a idade do paciente em exames de tórax.

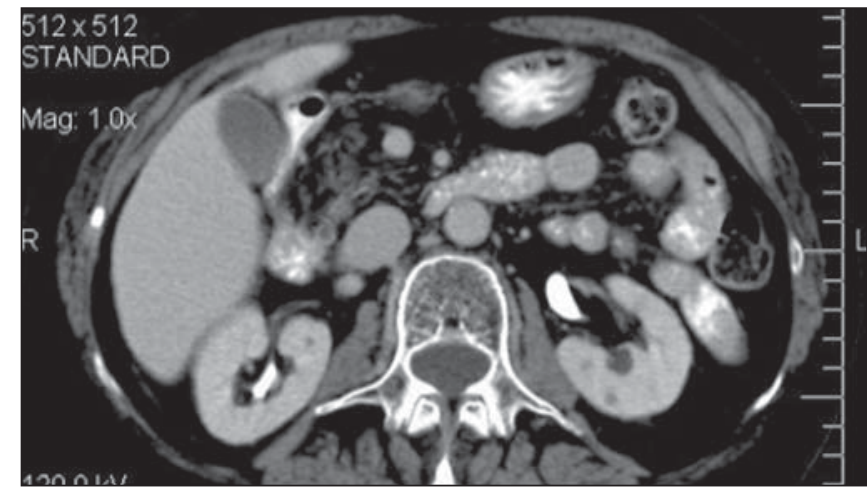

B

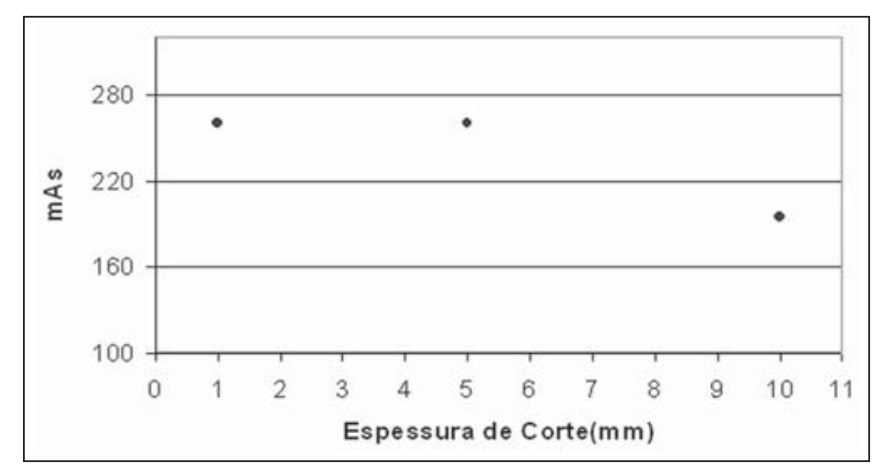

Figura 8. Relação entre o mAs utilizado e a espessura de corte em exames de tórax. 

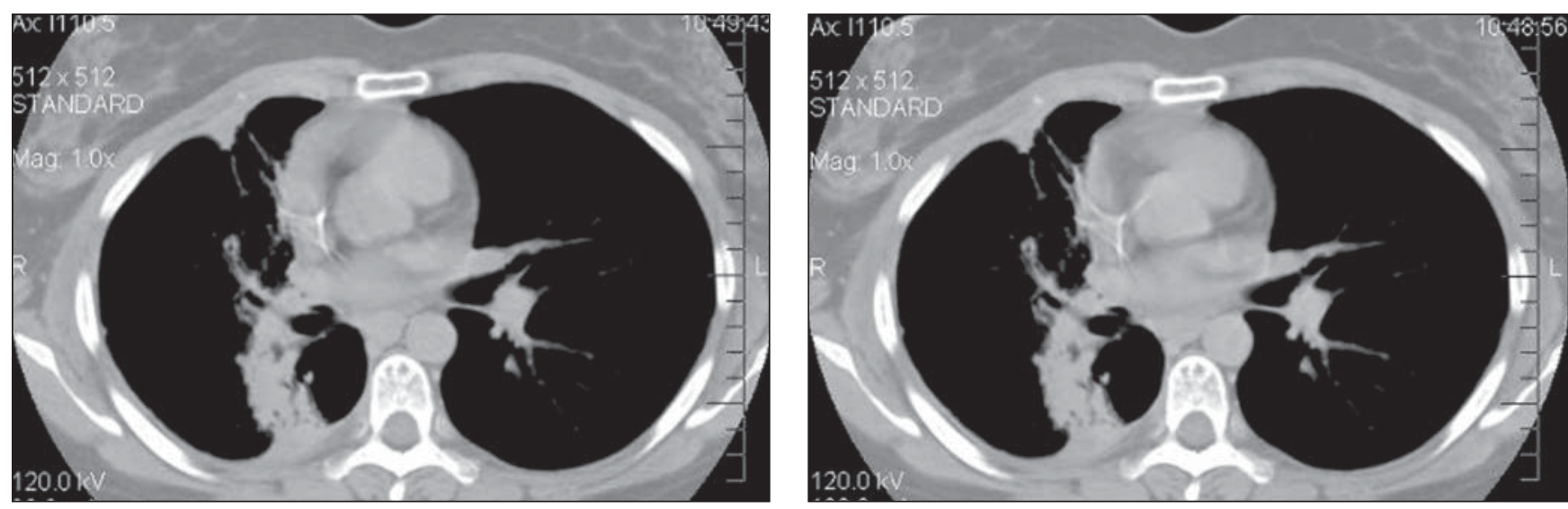

A

B

Figura 9. Imagens do tórax com 5 mm de espessura de corte. A: Imagem obtida com 120 mAs; B: Imagem obtida com 260 mAs.

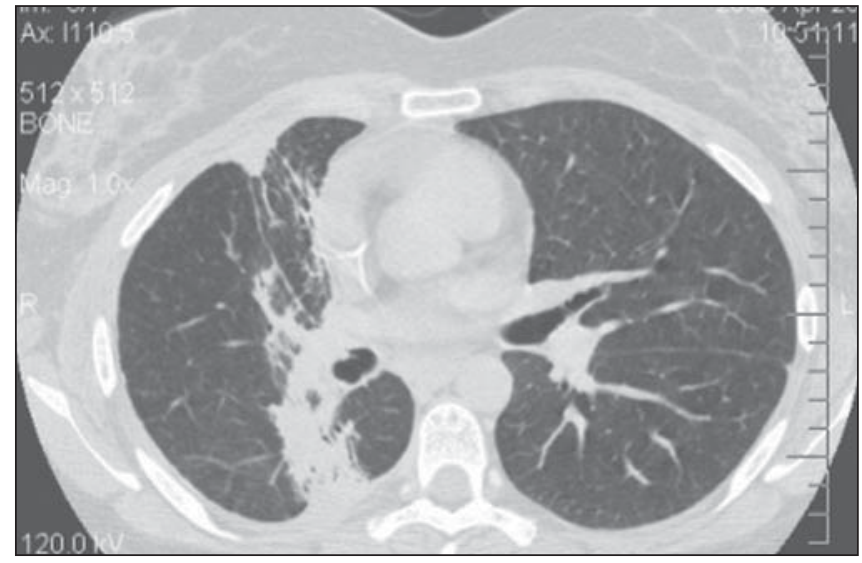

A

Figura 10. Imagens do tórax com 1 mm de espessura de corte. A: Imagem obtida com 150 mAs; B: Imagem obtida com 260 mAs.

gerida no mAs utilizado estão mostradas na Tabela 4

Os valores de MSAD para crianças estão subestimados, uma vez que foram utilizados os mesmos simuladores de adultos. Entretanto, a redução relativa na dose pôde ser usada para comparação.

A redução no aquecimento do tubo de raios $\mathrm{X}$ foi estimada apenas para pacientes adultos, sendo considerado o mAs médio de todos os exames avaliados. Esta redução foi de aproximadamente $13 \%$.

\section{Exames de abdome superior}

A Tabela 5 demonstra o resultado das medidas de MSAD e a redução porcentual entre os protocolos.

Para este exame a redução no aquecimento do tubo de raios $\mathrm{X}$ foi estimada considerando o mAs médio entre as três técnicas avaliadas. Esta redução foi de aproximadamente $23 \%$.

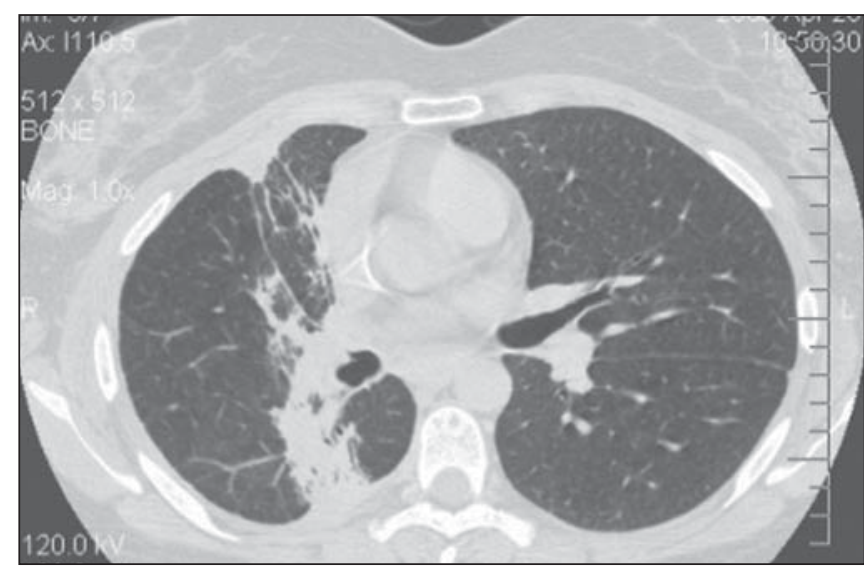

Tabela 4 Comparação e redução porcentual na MSAD para exames de crânio.

\begin{tabular}{|c|c|c|c|c|c|c|}
\hline Pacientes & Região & $\begin{array}{c}\text { mAs } \\
\text { utilizado }\end{array}$ & $\begin{array}{l}\text { MSAD } \\
\text { (mGy) }\end{array}$ & $\begin{array}{c}\text { mAs } \\
\text { sugerido }\end{array}$ & $\begin{array}{l}\text { MSAD } \\
\text { (mGy) }\end{array}$ & $\begin{array}{c}\text { Redução média } \\
\text { na dose }\end{array}$ \\
\hline \multirow{2}{*}{ Adultos } & Fossa posterior & 320 & 26,6 & 260 & 21,8 & \multirow{2}{*}{$20 \%$} \\
\hline & Supratentorial & 260 & 25,3 & 200 & 19,5 & \\
\hline \multirow{2}{*}{ 5-10 anos } & Fossa posterior & 260 & 21,8 & 240 & 20,0 & \multirow{2}{*}{$6 \%$} \\
\hline & Supratentorial & 200 & 24,4 & 195 & 23,7 & \\
\hline \multirow{2}{*}{$3-5$ anos } & Fossa posterior & 260 & 21,8 & 200 & 16,8 & \multirow{2}{*}{$13 \%$} \\
\hline & Supratentorial & 200 & 24,4 & 195 & 23,7 & \\
\hline \multirow{2}{*}{$1-3$ anos } & Fossa posterior & 260 & 21,8 & 195 & 16,3 & \multirow{2}{*}{$22 \%$} \\
\hline & Supratentorial & 200 & 24,4 & 160 & 19,6 & \\
\hline \multirow{2}{*}{6 meses- 1 ano } & Fossa posterior & 260 & 21,8 & 150 & 17,7 & \multirow{2}{*}{$29 \%$} \\
\hline & Supratentorial & 200 & 24,4 & 120 & 14,7 & \\
\hline \multirow{2}{*}{ 0-6 meses } & Fossa posterior & 260 & 21,8 & 120 & 14,0 & \multirow{2}{*}{$45 \%$} \\
\hline & Supratentorial & 200 & 24,4 & 90 & 11,0 & \\
\hline
\end{tabular}

Tabela 5 Comparação e redução porcentual na MSAD para exames de abdome superior.

\begin{tabular}{|l|c|c|c|c|c|}
\hline Peso(kg) & mAs utilizado & MSAD (mGy) & mAs sugerido & MSAD (mGy) & Redução média na dose \\
\hline$<50$ & 320 & 14,1 & 200 & 8,9 & $37 \%$ \\
50 a 80 & 320 & 14,1 & 260 & 11,6 & $18 \%$ \\
$>80$ & 320 & 14,1 & 320 & 14,1 & $0 \%$ \\
\hline
\end{tabular}


Tabela 6 Comparação e redução porcentual na MSAD para exames de tórax.

\begin{tabular}{|l|c|c|c|c|c|}
\hline \multicolumn{1}{|c|}{ Aquisição } & $\begin{array}{c}\text { mAs } \\
\text { utilizado }\end{array}$ & $\begin{array}{c}\text { MSAD } \\
\text { (mGy) }\end{array}$ & $\begin{array}{c}\text { mAs } \\
\text { sugerido }\end{array}$ & $\begin{array}{c}\text { MSAD } \\
\text { (mGy) }\end{array}$ & $\begin{array}{c}\text { Redução média } \\
\text { na dose }\end{array}$ \\
\hline Rotina seqüencial & 260 & 16,6 & 120 & 7,6 & $54 \%$ \\
Helicoidal & 195 & 19,7 & 120 & 10,9 & $45 \%$ \\
Alta resolução & 260 & 2,3 & 150 & 1,1 & $52 \%$ \\
\hline
\end{tabular}

\section{Exames de tórax}

A Tabela 6 demonstra o resultado das medidas de MSAD e a redução porcentual entre os protocolos.

A redução no aquecimento do tubo de raios $\mathrm{X}$ foi estimada considerando o $\mathrm{mAs}$ médio de todos os exames avaliados. Esta redução foi de aproximadamente $41 \%$.

\section{DISCUSSÃO}

A Portaria 453/98 do Ministério da Saúde ${ }^{(14)}$ apresenta níveis de referência para TC em paciente adulto típico apenas nos exames de crânio, coluna lombar e abdome. Os valores de MSAD para crânio e abdome, exames discutidos neste trabalho, são de, respectivamente, 50 e 25 mGy. Já o guia europeu ${ }^{(15)}$ apresenta níveis de referência para muitos exames, e os valores para crânio, abdome e tórax são de 60 , 35 e 30 mGy, respectivamente. Através das medidas realizadas conclui-se que os exames de rotina podem ser realizados com valores abaixo dos apresentados. Tanto na Portaria 453/98 quanto no guia europeu não são referenciados valores de MSAD para pacientes pediátricos, mas através da redução nas técnicas propostas, estes podem ser mantidos muito abaixo em relação aos níveis considerados para adultos. Os médicos radiologistas que avaliaram as imagens concordaram que um nível de ruído levemente maior não impede que o diagnóstico seja feito, mantendo-se, assim, a dose de radiação tão baixa quanto razoavelmente exeqüível (princípio ALARA) e desgastando menos o tubo de raios $\mathrm{X}$.

São muitos os trabalhos publicados sobre redução de dose no paciente em exa- mes de TC. Entretanto, não podemos falar o mesmo sobre aumento na vida útil do tubo de raios X. A literatura apresentada pelos fabricantes relaciona diretamente o desgaste do tubo de raios $\mathrm{X}$ com o aquecimento. Dessa forma, se considerarmos uma relação direta entre aquecimento e desgaste, pode-se calcular o número de exames a mais que será realizado com o mesmo tubo se forem adotadas as alterações sugeridas neste trabalho. Para os exames de crânio, que representam 20,6\% da totalidade, foi obtida redução de aproximadamente $13 \%$ no aquecimento. A vida útil média dos tubos deste equipamento foi de 204.480 exposições, segundo anotações do Serviço de Física Médica, em um total de 11 trocas de tubos. Dessa forma, poderiam ser realizadas mais 5.476 exposições, resultando em 322 exames com o mesmo tubo. Para os exames de abdome superior, que representam $20,4 \%$ da totalidade, a redução em $23 \%$ no aquecimento traria um aumento de 3.463 exposições, resultando em 479 exames, e para o exame de tórax, se for considerada redução média de $41 \%$ para as três técnicas utilizadas, conclui-se que esta traria um aumento de 506 exames com o mesmo tubo de raios X. Se for levado em consideração o valor de cada exame, com esse aumento pode-se reverter para o serviço o valor de uma ampola em alguns meses.

Portanto, é possível concluir que uma alteração nos protocolos dos exames descritos, visando uma padronização e redução do mAs utilizado nesses exames, trará uma redução significativa na dose recebida pelos pacientes sem comprometer a segurança diagnóstica. Também se conclui que essas alterações poderão trazer um aumento na vida útil do tubo de raios $\mathrm{X}$, reduzindo os custos da instituição.

\section{Agradecimentos}

Agradecemos a todos os técnicos em tomografia computadorizada e aos médicos radiologistas que contribuíram para o sucesso deste trabalho.

\section{REFERÊNCIAS}

1. Khorasani R, Goel PK, Ma'luf NM, Fox LA, Seltzer SE, Bates DW. Trends in the use of radiology with inpatients: what has changed in a decade? AJR 1998;170:859-61.

2. Shrimpton PC, Wall BF, Hart D. Diagnostic medical exposures in the U.K. Appl Radiat Isot 1999;50: 261-9.

3. Shrimpton PC, Edyvean S. CT scanner dosimetry. Br J Radiol 1998;71:1-3.

4. Huda W, Atherton JV, Ware DE, Cumming WA. An approach for the estimation of effective radiation dose at CT in pediatric patients. Radiology 1997; 203:417-22.

5. Judy PF. Evaluating computed tomography image quality. In: Goldman LW, Fowlkes JB, eds. Medical CT and ultrasound: current technology and applications. Madison, WI: Advanced Medical Publishing, 1995:359-78.

6. Ende JF, Huda W, Ros PR, Litwiller AL. Image mottle in abdominal CT. Invest Radiol 1999;34: 282-6.

7. Schreiber P. Heat management in $x$-ray tubes. Medica Mundi 1990;35:49-56.

8. Ravenel JG, Scalzetti EM, Huda W, Garrisi W. Radiation exposure and image quality in chest $\mathrm{CT}$ examinations. AJR 2001;177:279-84.

9. Naidich DP, Marshall CH, Gribbin C, Arams RS, McCauley DI. Low-dose CT of the lungs: preliminary observations. Radiology 1990;175:729-31.

10. Mayo JR, Whittall KP, Leung AN, et al. Simulated dose reduction in conventional chest $\mathrm{CT}$ : validation study. Radiology 1997;202:453-7.

11. Donnelly LF, Emery K-H, Brody AS, et al. Minimizing radiation dose for pediatric body applications of single-detector helical CT: strategies at a large Children's Hospital. AJR 2001;176:303-6.

12. Programa eFilm Workstation. Disponível em: URL: http://www.efilm.ca/. Acessado em janeiro de 2002.

13. American Association of Physicists in Medicine. Phantoms for performance evaluation and quality assurance of CT scanners. AAPM Report No. 1. 1977.

14. Brasil. Ministério da Saúde. Diretrizes de proteção radiológica em radiodiagnóstico médico e odontológico. Portaria no 453. Brasília, DF: Diário Oficial da União, 2/6/1998.

15. European guidelines on quality criteria for computed tomography. EUR 16262. May 1999. 